\title{
TERAPIA A LASER NO TRATAMENTO DE ÚLCERA DE PRESSÃO: REVISÃO INTECRATIVA
}

\section{LASER THERAPY IN PRESSURE ULCER TREATAMENT: AN INTEGRATIVE REVIEW}

Cleidenice dos Santos Orssatto(ORCID: 0000-0003-4377-252) ${ }^{1}$

Isadora Martins de Souza (ORCID: 0000-0002-1274-1389) ${ }^{1}$

Cíntia de Oliveira Cardoso(ORCID: 0000-0003-1015-249X)

Clediane Molina de Sales (ORCID: 0000-0002-4609-8643) ${ }^{1}$

${ }^{1}$ Curso de Fisioterapia, Faculdade de Educação e Meio Ambiente

Autor correspondente:

Cleidenice dos Santos Orssatto E-mail: cleidenice.orssatto@gmail. com

\begin{abstract}
RESUMO
O objetivo foi integrar dados de estudos experimentais e não experimentais que fundamentem o uso do Laser de Baixa Intensidade (LBI) na cicatrização de úlceras de pressão (UP) e identificar o tipo de LBI mais eficaz na cicatrização dessas lesões. Tratou-se de uma revisão de literatura do tipo integrativa, realizada por meio das seguintes plataformas: PEDro; SciELO; Lilacs; PubMed; Medline; catálogo da Capes e livros do acervo físico (biblioteca Júlio Bordignon) e virtual da faculdade Faema. Os descritores indexados ao DeCS utilizados foram: lesão por pressão; laser; terapia a laser; ferimentos e lesões, e suas variantes na língua inglesa. Foram inclusos estudos experimentais e não experimentais disponíveis na íntegra, publicados em português, espanhol e inglês, com temática vinculada ao uso do LBI como recurso principal para o tratamento de UP. Foram excluídos estudos que abordavam o uso do LBI no tratamento de outros tipos de feridas ou cujo recurso não era o principal meio de tratamento, além disso, foram excluídos estudos que se repetiam nas bases de dados. Foram recuperados 32 trabalhos, e após a aplicação dos critérios de inclusão e exclusão, 13 foram considerados elegíveis para inclusão nesta revisão. Esses trabalhos foram publicados entre os anos 2003 e 2018 e avaliaram os LBI com os seguintes meios ativos e diversos comprimentos de onda: AlGaInP, AlGaInP, GaAs, GaAlAs e HeNe. A maioria dos estudos encontrou evidências que embasam o uso do LBI no tratamento de UP. Sugere-se que, dos tipos de LBI, o de GaAlAs com comprimento de onda de 658 $\mathrm{nm}$ é o mais efetivo no tratamento de UP. Pontua-se a necessidade de estudos padronizados, a fim de chegar a um consenso definitivo.
\end{abstract}

Palavras-chave: Úlcera de pressão; Laser de baixa intensidade; Cicatrização

\begin{abstract}
The aim of this study was to integrate data from experimental and non-experimental studies that support the use of Low Intensity Laser (LIL) in pressure ulcer healing (PU) and to identify the type of LIL that is most effective in healing these lesions. This was an integrative literature review, performed through the following platforms: PEDro; SciELO; LILACS; PubMed; Medline; Capes catalog and books from the physical collection (Júlio Bordignon library) and from the Faema college. The DeCS indexed descriptors used were: pressure injury; laser; laser therapy; wounds and injuries and their variants in the English language. Experimental and non-experimental studies available in the full, published in Portuguese, Spanish, and English, with thematic linked to the use of LIL as the main resource for PU treatment were included. Studies that addressed the use of LIL in the treatment of other types of wounds or whose use was not the main means of treatment were excluded, and studies that were repeated in the databases were excluded. 32 papers were retrieved, and after applying the inclusion and exclusion criteria, 13 were considered eligible for inclusion in this review. These works were published between 2003 and 2018 and evaluated the LIL with the following active media and various wavelengths: AlGaInP, AlGaInP, GaAs, GaAlAs and HeNe. Most studies have found evidence to support the use of LIL and the treatment of PU. Of the LIL types, it is suggested that the $658 \mathrm{~nm}$ wavelength GaAlAs is the most effective in the treatment of PU. There is a need for standardized studies in order to reach a definite consensus.
\end{abstract}




\section{INTRODUÇÃO}

A pele é o maior órgão sensorial do corpo humano e funciona como revestimento do organismo, isolando do meio externo os demais componentes orgânicos. Ela possui funções vitais que a tornam indispensável à vida, a saber: regular a temperatura corporal; oferecer barreira contra agressões do meio ambiente; secretar e excretar água e metabólitos; receber estímulos sensitivos; oferecer defesa contra microrganismos; entre outras ${ }^{1-3}$.

Segundo Dardengo et al. ${ }^{4}$, a integridade da pele pode ser afetada por diversos mecanismos fisiopatológicos e/ou degenerativos, os quais podem repercutir no surgimento de úlceras de pressão (UP) ou lesões por pressão (LPP). Essas lesões afetam a pele e/ou tecidos subjacentes e ocorrem quando uma pressão ou uma pressão associada à fricção e/ou cisalhamento é exercida de forma contínua sobre determinada área, geralmente, sobre proeminências ósseas ${ }^{1,5}$.

Outros termos são utilizados para se referir às UP, como úlcera de decúbito ou escara. O termo decúbito, derivado do latim "decumbere", significa "deitado" e não engloba a ocorrência de lesão com o paciente em sedestação. O termo escara, por sua vez, refere-se somente ao tecido necrosado que pode recobrir a úlcera. Portanto, esses termos são inapropriados conceitualmente para se referir às UP ${ }^{5}$.

As UP são lesões recorrentes, incapacitantes e que afetam de forma significativa a qualidade de vida dos acometidos e de seus familiares, ao provocar dor, sofrimento e aumentar o tempo de internação. Além do impacto direto ao paciente, as UP também são onerosas ao sistema de saúde e estão associadas a elevados índices de mortalidade e morbidade ${ }^{6,7}$.

O principal fator de risco para o desenvolvimento de UP é a pressão, no entanto, fatores intrínsecos e extrínsecos se relacionam para determinar seu surgimento, tais como: internações prolongadas, mobilidade reduzida, má oxigenação, infecção, desnutrição, imobilidade, inatividade, desidratação, deficit neurológico, sudorese, anemia, extremos de idade, comorbidades, emagrecimento, obesidade, incontinência fecal e urinária, tabagismo, doença vascular, hipoproteinemia, edema localizado, sedação, umidade, cirurgias e polifarmácia ${ }^{7-9}$.

Segundo a National Pressure Ulcer Advisory Panel (NPUAP) ${ }^{10}$, as UP são classificadas em 4 estágios, a saber, Estágio 1: pele íntegra com eritema não branqueável; Estágio 2: perda de espessura parcial da pele com exposição da derme; Estágio 3: perda total da espessura da pele (o tecido adiposo é visível na úlcera); Estágio 4: perda total da espessura da pele e perda tissular. A NPUAP ${ }^{10}$ ainda elenca o tipo de UP não estadiável, que é caracterizada pela perda total da espessura da pele associada à perda tissular não visível, devido à presença esfacelo ou escara.

Diversos recursos são dispendidos para o tratamento das UP, como procedimento cirúrgico, curativos especiais e agentes biofísicos. No âmago dos agentes biofísicos, destaca-se o Laser de Baixa Intensidade (LBI), que tem sido apontado pela literatura como favorável à cicatrização de feridas, em especial nas resistentes ao tratamento convencional ${ }^{8,11}$.

O termo laser é uma abreviação do termo Light Amplification by Stimulated Emission of Radiation. Eles são denominados a partir do tipo de meio ativo utilizado, por exemplo, AsGa (arsênio-gálio) ou $\mathrm{HeNe}$ (hélio-neônio). O meio ativo pode ser um líquido, um sólido ou um gás cuja composição contenha íons, moléculas ou átomos capazes de armazenar energia e que, sob estímulo, liberam energia luminosa ${ }^{12}$.

Os lasers terapêuticos são recursos que produzem uma luz monocromática altamente refinada na faixa ultravioleta, visível ou não (infravermelha), capazes de provocar alterações fisiológicas nos tecidos. A potência da laserterapia de baixa intensidade é relativamente baixa (inferior a $500 \mathrm{~mW}$ ) e utiliza dosagens inferiores a 
$35 \mathrm{~J} / \mathrm{cm} 2$, as quais não provocam aquecimento detectável nos tecidos-alvos ${ }^{12,13}$.

A estimulação por LBI produz uma série de efeitos benéficos nos tecidos, como: melhoria da qualidade da cicatrização, estímulo à microcirculação, efeitos analgésicos, anti-inflamatórios, antiedematosos e antibactericida. Esses efeitos ocorrem devido ao incremento da produção de Adenosina Trifosfato (ATP), que proporciona aumento do número de mitoses e de síntese proteica, e, também, devido ao maior suprimento de nutrientes que favorece a angiogênese e a multiplicação celular ${ }^{3,14,15}$.

Considerando os efeitos benéficos que o LBI provoca no tecido, este estudo teve como objetivo integrar dados de estudos experimentais e não experimentais que fundamentem o uso desse recurso na cicatrização de UP, bem como identificar o tipo de LBI mais eficaz no tratamento dessas lesões.

\section{MÉTODOS}

Este estudo trata-se de uma revisão de literatura do tipo integrativa. Para o levantamento de dados, foram utilizados os seguintes descritores indexados ao Descritores em Ciências da Saúde (DeCS), seus respectivos sinônimos e associações: "lesão por pressão"; "laser"; "terapia a laser"; "ferimentos e lesões". Do mesmo modo, foram utilizados os descritores em inglês: "pressure ulcer"; "laser"; "laser therapy"; "wounds and Injuries".

Para as pesquisas, foram utilizadas as seguintes bases de dados eletrônicas: Physiotherapy Evidence Database (PEDro); Scientific Electronic Library Online (SciELO); Literatura latino-americana e do Caribe em Ciências da Saúde (Lilacs); US National Library of Medicine (PubMed); Medical Literature Analysis and Retrieval System Online (Medline). O levantamento de dados também foi realizado em repositórios de teses e dissertações e no Catálogo de Teses e Dissertações da Coordenação de Aperfeiçoamento de Pessoal de Nível Superior (Capes). Igualmente, foram consultados livros do acervo físico (biblioteca Júlio Bordignon) e virtual da faculdade Faema.
Foram elegíveis para inclusão estudos experimentais e não experimentais disponíveis integralmente, publicados em português, espanhol e inglês, com temática atrelada ao uso do LBI no tratamento de UP. Adotaram-se, como critérios de exclusão, abordagem do uso LBI no tratamento de outros tipos de feridas, abordagem do uso de outras modalidades fisioterapêuticas como recurso principal no tratamento de LPP e artigos repetidos nas bases de dados.

Por meio da consulta nas bases de dados, foram recuperados 32 trabalhos para leitura dos resumos. Após esse processo, aplicando-se os critérios de inclusão e exclusão, 13 trabalhos foram selecionados para leitura integral (figura 1).

Figura 1. Fluxograma do processo de seleção dos trabalhos

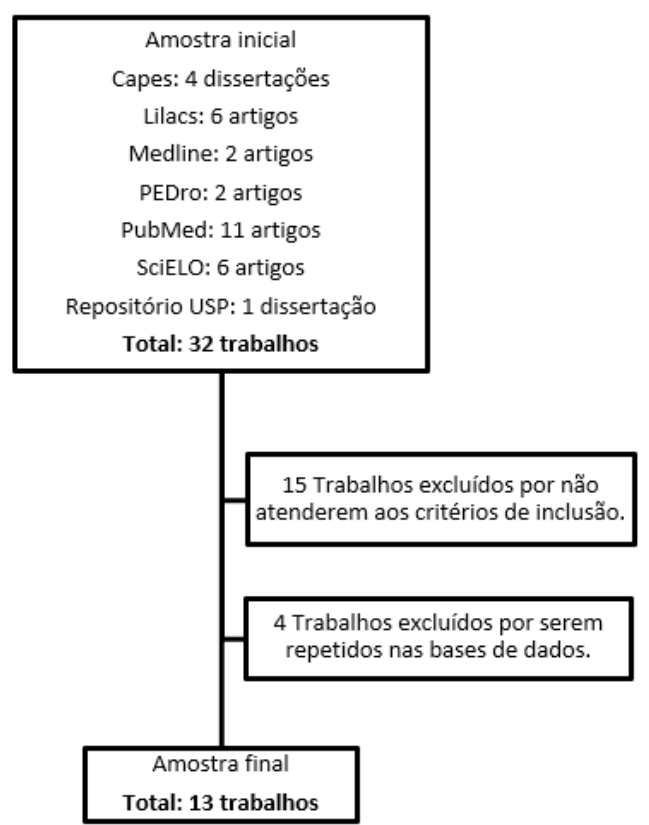

Para facilitar a análise e a síntese dos trabalhos, foi construído um quadro sinóptico constituído pelos seguintes itens: título do trabalho; nome dos autores; intervenção aplicada; resultados; conclusões. 


\begin{tabular}{|c|c|c|c|c|c|}
\hline \multirow{9}{*}{ 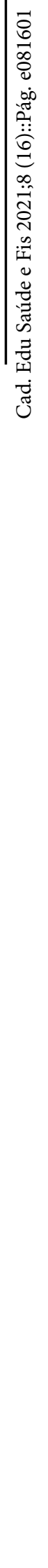 } & \multicolumn{5}{|c|}{ Quadro 1 - Síntese dos trabalhos inclusos na revisão integrativa } \\
\hline & \begin{tabular}{|l} 
Titulo do trabalho \\
\end{tabular} & Autores & \begin{tabular}{|l|l|} 
Intervenção aplicada \\
\end{tabular} & Resultados & \begin{tabular}{|l|} 
Conclusöes \\
\end{tabular} \\
\hline & $\begin{array}{l}\text { Efeitos do laser de baixa potência } \\
\text { na cicatrização de úlceras por } \\
\text { pressão em neonatos }\end{array}$ & Arruda AJA & $\begin{array}{l}\text { Avaliação dos efeitos do LBP na } \\
\text { cicatrização de UP em neonatos. A } \\
\text { amostra foi dividida aleatoriamente } \\
\text { nos grupos (n=10): G1 - tratamento } \\
\text { convencioonal com placa de } \\
\text { hidrocoloide, trocada a cada três } \\
\text { dias e } G 2 \text { - tratamento com laser } \\
\text { de diodo, em } 660 \text { nm, contato, em } \\
\text { média seis pontos por lesão, com } 5 \\
\text { mW, } 1,25 \mathrm{~J} / \mathrm{cm} 2 \text { por ponto, duas } \\
\text { vezes por semana. }\end{array}$ & $\begin{array}{l}\text { Já no primeiro dia dos } \\
\text { tratamentos, houve uma } \\
\text { redução de } 51 \% \text { na média das } \\
\text { áreas das úlceras quando } \\
\text { comparadas às úlceras tratadas } \\
\text { convencionalmente. Entretanto, } \\
\text { ambos os tratamentos } \\
\text { mostraram significante } \\
\text { efetividade na cicatrizacãa das } \\
\text { úlceras nos tempos avaliados. } \\
\end{array}$ & $\begin{array}{l}\text { A terapia com LBP mostrou } \\
\text { eficácia na cicatrizaçãoo das } \\
\text { UP em neonatos. }\end{array}$ \\
\hline & $\begin{array}{l}\text { Efeitos dos lasers Hélio-Neônio } \\
\text { (HeNe) e Arseneto de Gálio (AsGa) } \\
\text { associados à educacãáo em saúde } \\
\text { com foco na promoçáo da saúde de } \\
\text { portadores de úlcera por pressão }\end{array}$ & $\begin{array}{l}\text { Fialho LMF, } \\
\text { Baron VM, } \\
\text { Brandenburg C, } \\
\text { Martin ABTS }\end{array}$ & $\begin{array}{l}\text { Foram tratados um usuário com o } \\
\text { laser Hélio-Neônio (HeNe) (52 } \\
\text { sessõos) e dois com o laser } \\
\text { Arseneto de Gálio (AsGa) (22 e } 46 \\
\text { sessõos), selecionados } \\
\text { aleatoriamente, além do laser foi } \\
\text { realizado um trabalho preventivo } \\
\text { com eles. }\end{array}$ & \begin{tabular}{|l} 
A mensuração e o registro \\
fotográfico das feridas \\
demostram que houve \\
progresso significativo, com \\
cicatrização completa dos \\
processos ulcerativos de dois \\
usuários (O tratado com HeNe \\
em 52 sessões e um tratado \\
com AsGa com 22 sessões), \\
apenas um sujeito não \\
apresentou melhora da \\
cicatrização, constatou-se que \\
esse nâo seguia as orientações \\
de prevenção e abandonou o \\
tratamento a laser (foram \\
realizadas 46 sessôres).
\end{tabular} & $\begin{array}{l}\text { Os lasers de HeNe e AsGa } \\
\text { apresentaram-se eficazes } \\
\text { no processo de } \\
\text { cicatrização. Os usuários } \\
\text { que seguiram as } \\
\text { orientaçóes e cuidados de } \\
\text { prevenção obtiveram } \\
\text { melhores resultados do } \\
\text { que aqueles que não os } \\
\text { seguiram. }\end{array}$ \\
\hline & $\begin{array}{l}\text { Fechamento de úlceras de pressão } \\
\text { em pacientes com lesão medular. } \\
\text { proposta terapêutica }\end{array}$ & $\begin{array}{l}\text { Joppert D, } \\
\text { Bastos VH, } \\
\text { Machado D, } \\
\text { Nunes T, } \\
\text { Silveira LM, } \\
\text { Kale Junior N, } \\
\text { Goulart R, } \\
\text { Mello M, } \\
\text { Silva JG, } \\
\text { Orsini M }\end{array}$ & $\begin{array}{l}\text { O protocolo foi baseado no } \\
\text { atendimento multidisciplinar para } \\
\text { facilitar o fechamento de UP em } 5 \\
\text { lesados medulares de diferentes } \\
\text { causas e niveis neurológicos. A } \\
\text { fisioterapia utilizou laser } \\
\text { terapêutico (média de } 13 \text { sessões), } \\
\text { enquanto a enfermagem realizou } \\
\text { curativos com polihexanida e } \\
\text { hidrogel. }\end{array}$ & $\begin{array}{l}\text { Ocorreu piora em um caso } \\
\text { decorrente de complicaçōes } \\
\text { associadas e melhorias em } \\
\text { quatro casos, sendo que em } \\
\text { dois desses a ferida foi } \\
\text { totalmente fechada. }\end{array}$ & \begin{tabular}{|l|} 
O protocolo aplicado foi \\
efetivo quando seguido \\
corretamente, visto que o \\
resultado de maior \\
eficiencia foi do paciente \\
que acumulava fatores \\
individuais mais \\
desfavoráveis, exceto \\
pelas condiç̧os de ordem \\
socioeconônica, cultural, \\
familiar e de suporte \\
técnico.
\end{tabular} \\
\hline & $\begin{array}{l}\text { Laser therapy in pressure ulcers: } \\
\text { evaluation by the Pressure Ulcer } \\
\text { Scale for Healing and Nursing } \\
\text { Outcomes Classification }\end{array}$ & $\begin{array}{l}\text { Palagi S, } \\
\text { Severo IM, } \\
\text { Menegon DB, } \\
\text { Lucena AF }\end{array}$ & $\begin{array}{l}\text { Aplicação do LBI de AIGalnP, com } \\
\text { um comprimento de onda de 660 } \\
\text { nm, com emissão de luz continua e } \\
\text { pulsada, três vezes na semana, } \\
\text { totalizando } 15 \text { aplicaçōes. A } \\
\text { analise dos dados foi por } \\
\text { estatística descritiva, considerando } \\
\text { os escores na Pressure Ulcer } \\
\text { Scale for Healing (PUSH) Nursing } \\
\text { Outcomes Classification (NOC). }\end{array}$ & $\begin{array}{l}\text { Houve uma diminuição } \\
\text { significativa do tamanho da UP. } \\
\text { As dimensõos reduziram de } 7 \\
\text { cm para } 1,5 \mathrm{~cm} \text { de } \\
\text { comprimento e de } 6 \mathrm{~cm} \text { para } \\
1,1 \mathrm{~cm} \text { de largura. Também foi } \\
\text { observado aumento do écido } \\
\text { de epitelização e de } \\
\text { granulaçâa, além da diminuiçãã } \\
\text { da secreção e do odor da } \\
\text { ferida. }\end{array}$ & $\begin{array}{l}\text { Concluiu-se que houve } \\
\text { melhora importante no } \\
\text { processo } \\
\text { de cicatrizacãão da UP } \\
\text { tratada com aplicacão do } \\
\text { laser, demonstrada pela } \\
\text { aplicação do resultado } \\
\text { Cicatrização de } \\
\text { Feridas: segunda intenção } \\
\text { da NOC, e da escala } \\
\text { PUSH, além da evidência } \\
\text { do reaistro fotográfico. }\end{array}$ \\
\hline & $\begin{array}{l}\text { Efficacy of Multiwavelength Light } \\
\text { Therapy in the Treatment of } \\
\text { Pressure Ulcers in Subjects With } \\
\text { Disorders of the Spinal Cord: A } \\
\text { Randomized Double-Blind } \\
\text { Controlled Trial }\end{array}$ & \begin{tabular}{|l} 
Arun TB, \\
Nair KPS, \\
Murali T, \\
John A
\end{tabular} & 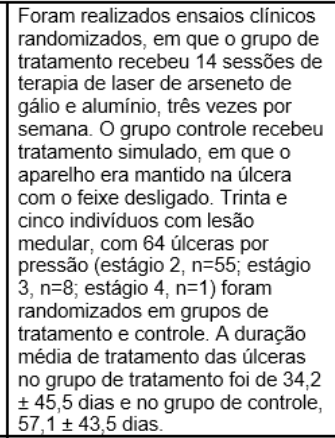 & $\begin{array}{l}\text { Não houve diferença } \\
\text { significativa na cicatrizacãa } \\
\text { entre o grupos des de tratamento e } \\
\text { controle. Dezoito úlceras no } \\
\text { grupo de tratamento e } 14 \text { no } \\
\text { grupo de controle cicatrizaram } \\
\text { completamente. Otempo médio } \\
\text { das úlceras para cicatrizar foi } \\
\text { de } 2,45 \pm 2,06 \text { semanas no } \\
\text { grupo de tratamento e de } 1,78 \pm \\
2,13 \text { semanas no grupo de } \\
\text { controle. }\end{array}$ & $\begin{array}{l}\text { Concluíram que o laser de } \\
\text { baixa potência de Arseneto } \\
\text { de Gálio e Alumínio oão } \\
\text { influenciou na cura das UP } \\
\text { em geral. Porém, } \\
\text { evidencias lieitadas } \\
\text { sugerem que melhorou a } \\
\text { cicatrização das UP nos } \\
\text { estágios } 3 \text { e } 4 \text {. }\end{array}$ \\
\hline & $\begin{array}{l}\text { Uso do laser de baixa intensidade } \\
\text { como adjuvante na cicatrizacão de } \\
\text { úlceras por pressãa em Unidade de } \\
\text { Cuidados Prolongados }\end{array}$ & Oliveira ER & $\begin{array}{l}\text { O autor avaliou a evolução e a } \\
\text { cicatrização de UP nos estágios II, } \\
\text { III e IV mediante a utilizaçăo da } \\
\text { LBP associada a } \\
\text { curativos de hidrogel com alginato. }\end{array}$ & $\begin{array}{l}\text { Todas as UP tratadas tiveram } \\
\text { redução significativa da área. } \\
\text { As UP de estágio III } \\
\text { apresentaram melhor evolução } \\
\text { na cicatrização do que as de } \\
\text { estágio IV. }\end{array}$ & $\begin{array}{l}\text { A laserterapia favoreceu a } \\
\text { cicatrização das UP nos } \\
\text { pacientes estudados. Os } \\
\text { estágios ea localização } \\
\text { das UP sãó fatores } \\
\text { importantes para a } \\
\text { cicatrização. }\end{array}$ \\
\hline & $\begin{array}{l}\text { Low-level laser therapy in the } \\
\text { treatment of pressure ulcers: } \\
\text { systematic review }\end{array}$ & $\begin{array}{l}\text { Machado RS, } \\
\text { Viana S, } \\
\text { Sbruzzi G }\end{array}$ & $\begin{array}{l}\text { Os autores efetuaram uma revisão } \\
\text { sistemática de estudos } \\
\text { randomizados objetivando avaliar } \\
\text { os efeitos do LBP em UP. }\end{array}$ & $\begin{array}{l}\text { O uso de LBP com } \\
\text { comprimento de onda de } 658 \\
\text { nm apresentou resultados } \\
\text { significativos na reduçăo das } \\
\text { UP. }\end{array}$ & \\
\hline
\end{tabular}




\begin{tabular}{|c|c|c|c|c|}
\hline $\begin{array}{l}\text { Laser photobiomodulation in } \\
\text { pressure ulcer healing of human } \\
\text { diabetic patients: gene expression } \\
\text { analysis of inflammatory } \\
\text { biochemical markers }\end{array}$ & $\begin{array}{l}\text { Ruh AC, } \\
\text { Frigo L, } \\
\text { Cavalcanti MFXB, } \\
\text { Svidnicki P, } \\
\text { Vicari VN, } \\
\text { Lopes-Martins RAB, } \\
\text { Leal Junior ECP, } \\
\text { Isla N, } \\
\text { Diomede F, } \\
\text { Trubiani O, } \\
\text { Favero GM }\end{array}$ & $\begin{array}{l}\text { Os autores avaliaram a expressão } \\
\text { gênica de fatores inflamatórios que } \\
\text { participam do processo de } \\
\text { cicatrização dos tecidos sob os } \\
\text { efeitos da LBP. }\end{array}$ & $\begin{array}{l}\text { O LBP promoveu melhora da } \\
\text { aparência macroscópica; } \\
\text { fatores de crescimento } \\
\text { relacionados à angiogênese } \\
\text { aumentaram. }\end{array}$ & $\begin{array}{l}\text { O LBP é uma ferramenta } \\
\text { de cura promissora para } \\
\text { reduzir o tamanho das UP. }\end{array}$ \\
\hline $\begin{array}{l}\text { Efeitos da laserterapia no } \\
\text { tratamento de lesões por pressão: } \\
\text { uma revisão sistemática }\end{array}$ & $\begin{array}{l}\text { Bernardes LO, } \\
\text { Jurado SR }\end{array}$ & $\begin{array}{l}\text { Os autores realizaram uma revisão } \\
\text { sistemática com o objetivo de } \\
\text { estudar a eficácia da LBI na } \\
\text { cicatrização de UP. }\end{array}$ & $\begin{array}{l}\text { Foram selecionados } 11 \text { artigos } \\
\text { científicos. Os LBI com } \\
\text { comprimento de onda de } 658 \\
\text { nm foram os mais eficazes no } \\
\text { tratamento das UP. }\end{array}$ & $\begin{array}{l}\text { O LBI apresentou-se como } \\
\text { um tratamento promissor } \\
\text { no que diz respeito à } \\
\text { aceleração da cicatrização } \\
\text { das UP. }\end{array}$ \\
\hline $\begin{array}{l}\text { Terapia a laser na cicatrização da } \\
\text { úlcera por pressão em adultos e } \\
\text { idosos: revisão sistemática }\end{array}$ & Petz FFC & $\begin{array}{l}\text { A autora realizou uma revisão } \\
\text { sistemática para avaliar a } \\
\text { efetividade e a segurança do LBP } \\
\text { na cicatrização de UP em } \\
\text { comparação com diferentes tipos } \\
\text { de LBP, terapias tópicas, placebos } \\
\text { e agentes fisicos em adultos e } \\
\text { idosos. }\end{array}$ & $\begin{array}{l}\text { Cinco estudos foram incluídos } \\
\text { na análise. O laser GaAlAs } \\
\text { com o parâmetro } 658 \mathrm{~nm} \text { foi o } \\
\text { que obteve significância } \\
\text { estatística no desfecho } \\
\text { cicatrização completa. A } \\
\text { redução da área foi semelhante } \\
\text { para os grupos experimentais e } \\
\text { de controle em todos os } \\
\text { estudos. }\end{array}$ & $\begin{array}{l}\text { Os efeitos produzidos pelo } \\
\text { laser foram semelhantes } \\
\text { aos do grupo controle em } \\
\text { relação à efetividade e } \\
\text { segurança. Os achados } \\
\text { dos ensaios clínicos } \\
\text { randomizados foram } \\
\text { insuficientes para avaliar a } \\
\text { efetividade da terapia a } \\
\text { laser. }\end{array}$ \\
\hline $\begin{array}{l}\text { Efficacy of low-level laser therapy in } \\
\text { the management of stage III } \\
\text { decubitus ulcers: a prospective, } \\
\text { observer blinded multicentre } \\
\text { randomised clinical trial }\end{array}$ & $\begin{array}{l}\text { Lucas C, } \\
\text { Gemert Van MJC, } \\
\text { Hann RJ }\end{array}$ & $\begin{array}{l}\text { Estudo clínico prospectivo, } \\
\text { randomizado e multicêntrico, cego } \\
\text { pelos observadores, com objetivo } \\
\text { de avaliar o efeito do LBP como } \\
\text { coadjuvante no tratamento UP. }\end{array}$ & $\begin{array}{l}\text { Participaram do estudo } 86 \\
\text { pacientes, sendo } 47 \text { sujeitos ao } \\
\text { tratamento consensual } \\
\text { predominante e } 39 \text { submetidos } \\
\text { ao tratamento com LBP } \\
\text { adicionalmente. }\end{array}$ & $\begin{array}{l}\text { Não foram encontradas } \\
\text { evidências que embasem o } \\
\text { uso do LBP como } \\
\text { coadjuvante no tratamento } \\
\text { de UP. }\end{array}$ \\
\hline $\begin{array}{l}\text { Tratamento de úlceras de decúbito } \\
\text { com laser } \\
\text { de baixa intensidade }\end{array}$ & \begin{tabular}{|l} 
Araujo AR, \\
Chaves MEA, \\
Tizon AFF, \\
Da Silva DB, \\
Piantino LL, \\
Azevedo VS \\
\end{tabular} & $\begin{array}{l}\text { Aplicação de LBP, durante seis } \\
\text { semanas, com duas aplicações } \\
\text { semanais. }\end{array}$ & $\begin{array}{l}\text { Aprimoramento da cicatrização } \\
\text { das UP. }\end{array}$ & $\begin{array}{l}\text { O LBP foi eficaz no } \\
\text { tratamento de UP crônicas. }\end{array}$ \\
\hline $\begin{array}{l}\text { Tratamento de úlcera de pressão } \\
\text { através do laser AsGa de } \\
904 \mathrm{~nm} \text { - um relato de caso }\end{array}$ & $\begin{array}{l}\text { Stefanello TD, } \\
\text { Hamerski CR }\end{array}$ & $\begin{array}{l}\text { Laser de } 904 \mathrm{~nm} \text {, com aplicação } \\
\text { pontual e de varredura, duas vezes } \\
\text { na semana, durante } 7 \text { semanas. }\end{array}$ & $\begin{array}{l}\text { Após as } 7 \text { semanas, houve } \\
\text { cicatrização completa da } \\
\text { úlcera. }\end{array}$ & $\begin{array}{l}\text { A terapia de LBP acelera a } \\
\text { cicatrização das UP. }\end{array}$ \\
\hline
\end{tabular}


Os 13 trabalhos que atenderam aos critérios de inclusão foram publicados entre os anos de 2003 e 2018. Entre eles, 10 são artigos científicos e 3 são dissertações de mestrado.

Quanto aos tipos de estudos incluídos nesta revisão, são: quatro estudos de caso, três revisões sistemáticas, três ensaios clínicos randomizados, um ensaio clínico não randomizado, uma análise comparativa e um estudo descritivo observacional.

Os estudos inclusos nesta revisão integrativa avaliaram os LBI com os seguintes meios ativos no tratamento de UP: Aluminum Gallium Arsenide Phosphide (AlGaInP), Indium-Galium-Aluminium phosphide (AlGaInP), Gallium Arsenide (GaAs), Galio Aluminioarsenieto (GaAlAs), Helium Neon (HeNe). Foram utilizados comprimentos de onda variados, que serão tratados mais adiante.

\section{DISCUSSÃO}

Os primeiros estudos envolvendo o uso do LBI no tratamento de úlceras ocorreram entre o final da década de 1960 e o início da década de 1970. Nesses estudos, os resultados advindos da utilização do laser de $\mathrm{HeNe}$, com doses de até $4 \mathrm{~J} / \mathrm{cm} 2$, foram positivos na redução da dor e melhora da cicatrização. Nas décadas posteriores, o uso desse recurso se mostrou promissor no tratamento de diversos tipos de lesões ulceradas, em especial daquelas crônicas e de complexa resolução ${ }^{16}$.

Apesar das evidências trazidas pelos estudos $^{3,17,18}$, ainda não existe um consenso sobre o tipo de laser e respectivo comprimento de onda mais efetivo na cicatrização de UP.

O laser de GaAs produz uma onda de luz entre 904 e $910 \mathrm{~nm}$, a qual é invisível ao olho humano (infravermelha) ${ }^{12}$. Nos estudos de Araújo et al. ${ }^{11}$, Stefanelo e Hamerski ${ }^{13}$ e Fialho et $a^{1.3}$, o laser GaAs foi utilizado com comprimento de onda de
904 nm, e o número de aplicações foi de, respectivamente, 12,14 e 22 . O estudo de Araújo et al. ${ }^{11}$ consistiu em um ensaio clínico randomizado, cuja amostra foi composta por dois indivíduos adultos. No caso um, observou-se que a UP passou de 3,4 $\mathrm{cm}$ para $1,5 \mathrm{~cm}$; e no caso dois, de $3,0 \mathrm{~cm}$ para 2,0 cm, sendo esta considerada uma redução significativa. No estudo de Stefanelo e Hamerski ${ }^{13}$, a UP cuja área inicial era de 19,5 cm, ao final das 14 sessões, obteve $100 \%$ de cicatrização. No estudo de Fialho et al. ${ }^{3}$, o laser de GaAs (904 nm) promoveu cicatrização completa da UP ao final das 22 aplicações.

O ensaio clínico randomizado de Lucas et al.19 também avaliou o uso do laser de GaAs com comprimento de onda de 904 $\mathrm{nm}$, porém com amostra significativamente maior que a dos estudos supracitados $(n=86)$. Neste estudo, o grupo experimental recebeu o tratamento consensual produzido e recomendado pela NPUAP, associado à aplicação de laser de GaAs com comprimento de onda de $904 \mathrm{~nm}$. O grupo controle recebeu apenas o tratamento consensual, que consiste em: limpeza das feridas, prestação de informações e instruções ao paciente, aplicação de curativos úmidos e mudança regular de decúbito. No grupo experimental, o LBI foi aplicado 5 vezes por semana, totalizando 30 aplicações. Neste estudo, não foram encontradas diferenças significativas entre o grupo experimental e o grupo controle, o que levou os autores a concluir que não existem evidências que embasem o uso do LBI como coadjuvante no tratamento de UP.

O laser de AlGaInP possui comprimento de onda de $685 \mathrm{~nm}$, atua no espectro visível da luz e seu modo de saída é contínuo. Comparado ao laser de HeNe, o laser de AlGaInP penetra mais profundamente os tecidos $^{20}$.

Os estudos de Ruh et al. ${ }^{21}$, Arruda ${ }^{22}$ e Palagi23 utilizaram o laser de AlGaInP no tratamento de UP, todos com comprimento de onda de $660 \mathrm{~nm}$. O estudo de Ruh et al. ${ }^{21}$ consistiu em um ensaio clínico randomizado e contou com uma amostra de 8 indivíduos adultos com UP. Após as 12 
aplicações do laser de AlGaInP $660 \mathrm{~nm}$, foi evidenciado que houve um aumento de $50 \%$ do tecido de granulação, de forma que os autores concluíram que o LBI é um recurso de cura promissor para redução do tamanho das UP.

No estudo de Arruda $^{22}$, o grupo experimental foi tratado com laser de AlGaInP $660 \mathrm{~nm}$, e no grupo controle foi realizado o tratamento convencional (lavagem da UP com solução fisiológica, secagem com gaze estéril e aplicação de hidrocoloide). A amostra foi composta por 20 neonatos. Logo no primeiro dia de tratamento, houve redução de $51 \%$ da área da UP tratadas com LBI em comparação ao grupo controle. No segundo dia, houve remissão total das UP tratadas com o LBI, demonstrando a eficácia desse recurso na cicatrização de UP em neonatos.

No estudo de Palagi ${ }^{23}$, o laser AlGaInP $660 \mathrm{~nm}$ foi aplicado 15 vezes em um paciente adulto internado em Unidade de Terapia Intensiva (UTI). Após o tratamento, foi verificada redução significativa da área da UP, passando do comprimento inicial de $7 \mathrm{~cm}$, para $1,5 \mathrm{~cm}$, e largura de $6 \mathrm{~cm}$ para $1,1 \mathrm{~cm}$.

O estudo de Oliveira ${ }^{24}$ também utilizou o comprimento de onda de $660 \mathrm{~nm}$ em seu ensaio clínico não randomizado, porém não informou o tipo de meio ativo do LBI utilizado. Participaram desse estudo 13 pacientes internados, com um total de 32 UP. Os pacientes foram divididos em grupo de tratamento e grupo controle, sendo o primeiro tratado com LBI e curativos de hidrogel com Alginato; e o segundo, apenas com o curativo. $O$ desfecho do estudo apontou para redução significativa de todas as UP do grupo tratado com LBI em comparação com o grupo controle.

O ensaio clínico randomizado de Arun et al. ${ }^{25}$ também utilizou o laser de AlGaInP, no entanto, o comprimento de onda utilizado foi de $820 \mathrm{~nm}$. A amostra foi composta por 35 adultos, com 64 UP. No estudo, foi associado o uso do laser com terapia de luz de diversos cumprimentos de onda, curativos e educação sobre o tratamento. $\mathrm{O}$ grupo controle recebeu terapia de luz simulada e curativo. Após as 14 sessões de tratamento, não foi evidenciada diferença significativa na cura da úlcera entre os grupos, no entanto, observou-se que a terapia de luz foi capaz de reduzir o tempo gasto para as UP de estágio 3 e 4, que atingiram o estágio 2.

O laser de HeNe produz luz visível com comprimento de onda de $632,8 \mathrm{~nm}$, por meio da mistura dos gases Hélio e Neônio12. Os estudos de Joppert et al. ${ }^{26}$ e de Fialho et al. ${ }^{3}$ avaliaram este tipo de LBI.

Joppert et al ${ }^{26}$ associaram o uso do laser de $\mathrm{HeNe}$ com comprimento de onda de 660 $\mathrm{nm}$ ao uso de curativo com polihexanida e hidrogel. O tratamento variou de 35 a 47 dias; e após esse período, 2 das 5 UP tratadas obtiveram fechamento completo.

Fialho et al. ${ }^{3}$, por sua vez, utilizaram o laser $\mathrm{HeNe}$ com comprimento de onda de $623,8 \mathrm{~nm}$. Além das 52 sessões de laserterapia, também foram fornecidas instruções educativas sobre o tratamento das UP, incluindo cuidados com higiene pessoal, alimentação, realização do curativo, utilização de dispositivos de alívio de pressão, entre outras. Conforme já mencionado anteriormente, no estudo de Arun et al. ${ }^{25}$, também foi observada a associação do LPP à educação em saúde.

Além dos LBI de $\mathrm{HeNe}$, AsGa e AlGaInP, as revisões sistemáticas incluíram ainda estudos que utilizaram o laser de GaAlAs no tratamento de UP. O laser de GaAlAs produz faixa de luz infravermelha, com comprimento de onda de 830 $\mathrm{nm}$. Esse meio ativo, no entanto, pode ser manipulado para produção diversos comprimentos de onda ${ }^{12}$.

A revisão sistemática de Bernardes e Jurado $^{15}$ avaliou estudos que utilizaram o LBI de diversos meios ativos ( $\mathrm{HeNe}$, AlGaInP, outros não especificados) e diversos comprimentos de onda $(658 \mathrm{~nm}$, $660 \mathrm{~nm}, 830 \mathrm{~nm}, 940 \mathrm{~nm}, 808 \mathrm{~nm}$, outros não especificados). Os autores concluíram que, entre os LBI utilizados, os com comprimento de onda de $658 \mathrm{~nm}$ são os mais eficientes no tratamento de UP, porém não especificaram o tipo de meio ativo.

A revisão sistemática de Machado e Sbruzzi $^{27}$, por sua vez, incluiu estudos que avaliaram o uso dos seguintes LBI no tratamento de UP: GaAs 904mn, GaAlAs 808mn, GaAlAs 648 nm, GaAlAs 658mn. Entre esses, concluiu-se que o LBI de GaAlAs 658mn apresentou resultados significativos.

Na revisão sistemática de $\mathrm{Petz}^{8}$, foram 
inclusos 5 ensaios clínicos randomizados, que avaliaram os seguintes tipos de LBI: GaAs 904mn, GaAlAs 980, GaAllPh 650mn, GaAlAs 808, GaAlAs 658mn, $820 \mathrm{mn}$ (meio ativo não informado). Alguns estudos avaliaram mais de um tipo de LBI. O estudo concluiu que, entre os LBI utilizados, o de GaAlAs com comprimento de onda de $658 \mathrm{~nm}$ obteve significância estatística quando comparado a laserterapia de baixa intensidade com comprimentos de onda superiores e cuidados padronizados.

Apesar da grande quantidade de trabalhos sobre o uso do LBI no tratamento de UP, a baixa qualidade metodológica, a não padronização e a utilização de amostras pequenas nos ensaios clínicos foram fatores que limitaram a realização deste estudo.

\section{CONSIDERAÇÕES FINAIS}

Os resultados obtidos nesta revisão integrativa são conflitantes, no entanto, observou-se que a maioria dos estudos encontrou evidências que embasam o uso do LBI no tratamento de UP. Também foi verificado que a associação entre o uso de LBI e o fornecimento de informações e instruções relacionadas com o tratamento das UP pode ser benéfico.

A heterogeneidade dos estudos quanto ao tipo meio ativo utilizado, parâmetros empregados e tempo de tratamento é fator que dificulta definir o tipo de LBI mais eficaz no tratamento de UP. Outra barreira para chegar a um consenso é a ausência de informações, em alguns estudos, quanto aos parâmetros utilizados na aplicação ou o tipo de LBI utilizado.

Todavia, cabe ressaltar que duas das revisões sistemáticas inclusas nesta revisão integrativa foram unânimes e categóricas ao afirmar que o tipo de LBI mais efetivo no tratamento de UP é o de GaAlAs com comprimento de onda de $658 \mathrm{~nm}$. A terceira revisão sistemática inclusa neste estudo também apontou para a maior efetividade do comprimento de onda de 658 $\mathrm{nm}$, porém não informou o tipo de LBI.
Considerando a confiabilidade e a validade das revisões sistemáticas, é possível sugerir que o tipo de laser com maior efetividade no tratamento de UP é o de GaAlAs com comprimento de onda de $658 \mathrm{~nm}$. No entanto, é importante que seja realizada a padronização dos estudos, a fim de chegar a um consenso definitivo e, consequentemente, possibilitar o desenvolvimento de protocolos de tratamento assertivos.

\section{REFERÊNCIAS}

1. Rivitti EA. Manual de dermatologia clínica de Sampaio e Rivitti. São Paulo: Artes Médicas; 2014.

2. Abrahamsohn, P. Histologia. Rio de Janeiro: Guanabara Koogan; 2016.

3. Fialho LMF, Baron VM, Brandenburg C, Martins ABTS. Efeitos dos lasers Hélio-Neônio (HeNe) e Arseneto de Gálio (AsGa) associados à educação em saúde com foco na promoção da saúde de portadores de úlceras por pressão. Rev Med Minas Gerais. 2017;(27):20-26.

4. Dardengo DD, Gonçalves DM, Pinto HLB, Valle LF. Avaliação do índice de conhecimento dos cuidadores de clientes portadores de úlcera por pressão em um bairro do município de Cachoeiro de Itapemirim. Revista Camiliana de Iniciação Científica. 2006;1(1).

5. Wada A, Neto NT, ferreira MC. Úlceras por pressão. Rev Med (São Paulo). 2010;89(3-4):170-177.

6. Silva DRA et al. Pressure ulcer dressings in critical patients: a cost analysis Rev Esc Enferm USP. 2017;(51):1-8.

7. Andrade CCD, Ribeiro AC, Carvalho CAS, Ruas CM, Borges EL. Ocorrência de úlcera por pressão e perfil epidemiológico e clínico dos pacientes internados em uma unidade hospitalar da Fundação Hospitalar de Minas Gerais. Rev Med Minas Gerais. 2018;28(Supl. 5):161-7.

8. Petz FFC. Terapia a laser na cicatrização da úlcera por pressão em adultos e idosos: revisão sistemática [dissertação]. 
Curitiba: Universidade Federal do Paraná; 2015.

9. Otto C, Schumacher B, Wiese LPL, Ferro C, Rodrigues RA. Fatores de risco para o desenvolvimento de lesão por pressão em pacientes críticos. Enferm Foco. 2019;10(1):07-11.

10. National Pressure Ulcer Advisory Panel (NPUAP). Pressure Ulcer Stages Revised [Internet]. Washington, 2016 [acessado 2019 nov 10]. Disponível em: https://npuap.org/page/ PressureInjuryStages.

11. Araújo AR, Chaves MEA, Tizon AFF, Da Silva DB, Azevedo VS. Tratamento de úlceras de decúbito com laser de baixa intensidade. Fisioterapia Bras. 2009;2(10):135-138.

12. Starkey C. Recursos terapêuticos em fisioterapia. 4. ed. Barueri, SP: Manole; 2017.

13. Stefanello TD, Hamerski CR. Tratamento de úlcera de pressão através do laser AsGa deE 904 nm - Um relato de caso. Arquivos de Ciências da Saúde da UNIPAR. 2006;10(2):99-103.

14. Assis GM; Moser AD de L. Laserterapia em úlceras por pressão: limitações para avaliação de resposta em pessoas com lesão medular. Texto Contexto - Enferm. 2013;22(3):850-856.

15. Bernardes LO, Jurado SR. Efeitos da laserterapia no tratamento de lesões por pressão: uma revisão sistemática. Revista Cuidarte. 2018;9(3):2423-2434.

16. Araújo AR, Chaves MEA, Leal BB, Marval CA, Pinotti M, Alves GES, et al. Efeitos do laser de baixa potência no tratamento de úlceras de pressão em um equino. Fisioterapia Bras. 2008;1(9):59-63.

17. Corrêa FI, Prado FS, Miranda CM, De Souza APG, Corrêa JCF. O uso do laser $\mathrm{HeNe}(638,8 \mathrm{~nm})$ no fechamento de feridas. Fisioterapia Bras. 2019;4(2):144-148.

18. Caetano AM. Custo-efetividade da laserterapia no tratamento de lesão por pressão [tese]. São Paulo: Universidade Nove de Julho; 2019.

19. Lucas C, Gemert Van MJC, Hann RJ.
Efficacy of low-level laser therapy in the management of stage III decubitus ulcers: a prospective, observer-blinded multicentre randomised clinical trial. Lasers Med Sci. 2003;(18):72-77.

20. Prockt AP, Takahashi A, Pagnancelli RM. Uso de terapia com laser de baixa intensidade na cirurgia bucomaxilofacial. Rev Port Estomatol Med Dent Cir Maxilofac. 2008;49(4):247-255.

21. Ruh AC, Frigo L, Cavalcanti MFXB, Svidnicki P, Vicari VN, Lopes-Martins $\mathrm{RAB}$, et al. Laser photobiomodulation in pressure ulcer healing of human diabetic patients: gene expression analysis of inflammatory biochemical markers. Laser Med Sci. 2018;33(1):165-171.

22. Arruda, AJ. Efeitos do laser de baixa potência na cicatrização de úlceras por pressão em neonatos [dissertação] São Paulo: Universidade Camilo Castelo Branco; 2014.

23. Palagi S, Severo IM, Menegon DB, Lucena AF. Laser therapy in pressure ulcers: evaluation by the Pressure Ulcer Scale for Healing and Nursing Outcomes Classification. Rev Esc Enferm da USP. 2015 49(5):820-826.

24. Oliveira ER. Uso do laser de baixa intensidade como adjuvante na cicatrização de úlceras por pressão em unidade de cuidados prolongados [dissertação]. Belo Horizonte: Instituto de Ensino e Pesquisa; 2013

25. Arun TB, Nair KPS, Murali T, John , A. Efficacy of Multiwavelength Light Therapy in the Treatment of Pressure Ulcers in Subjects With Disorders of the Spinal Cord: A Randomized Double-Blind Controlled Trial. Arch Phys Med Rehabil. 2004;85(10):1657-61.

26. Joppert D, Bastos VH, Machado D, Silveira LM, Kale Junior N, Goulart R, et al. Fechamento de úlceras de pressão em pacientes com lesão medular: proposta terapêutica. Fisioterapia Bras. 2011;12(4): 303-308.

27. Machado RS, Viana S, Sbruzzi G. Low-level laser therapy in the treatment of pressure ulcers: systematic review. Laser Med Sci. 2017;32(4):937-944.
Recebido: 05/12/2019

Aprovado: 26/11/2020 